Ann. Biol, anim, Bioch. Biophys, I975, $15(4), 62$ I-63.

\title{
AUGMENTATION DE L'EFFICACITÉ DE LA SPERMATOGENÈSE PAR L'HÉMICASTRATION CHEZ LE RAT ET LE BÉLIER
}

\author{
Marie-Thérèse HOCHEREAU de REVIERS \\ avec la collaboration technique de Christine Perreau \\ Station de Physiologie de la Reproduction, \\ Centre de Recherches de Tours, I. N.R. A., \\ Nouzilly, 37380 Monnaie.
}

\begin{abstract}
RÉSUMÉ
L'hémicastration pratiquée chez le Rat impubère ou prépubère ou chez le Bélier adulte entraîne une hypertrophie compensatrice du testicule restant.

Les cellules de Sertoli répondent à l'hémicastration par une augmentation numérique chez le Rat hémicastré à l'âge de $\mathrm{r}$ jour, ou un accroissement de la taille nucléaire chez le Bélier adulte.

Les spermatogonies souches sont augmentées après hémicastration chez le Bélier adulte. Le rendement des divisions spermatogoniales est amélioré après hémicastration chez le Rat de 5 jours.

Le rendement de l'ensemble des processus comprenant la prophase méiotique, les divisions méïotiques ct le début de la spermiogenèse est augmenté significativement chez le Rat hémicastré à 5 ou 10 jours. Chez le Bélier, cette étape est sensible à l'hémigonadectomie.

De l'ensemble de ces modifications, il découle que chez le Raton hémicastré à I ou 5 jours, ou chez le Bélier adulte, la production quotidienne de gamètes est significativement augmentée.

La fertilité des rats hémicastrés à l'âge cle 5 jours n'est pas inférieure à la normale.

Chez le Bélier adulte, la quantité d'hormone LH circulant dans le sang augmente significativement après hémicastration au printemps.
\end{abstract}

\section{INTRODUCTION}

Les dégénérescences cellulaires qui ont lieu tout au long de la spermatogenèse et plus particulièrement au cours des divisions spermatogoniales sont quantitativement très importantes et diminuent de façon drastique l'efficacité de la spermatogenèse (jusqu'à $66 \mathrm{p}$. Ioo de perte). Il est donc important de connaître les facteurs qui contrôlent les rendements des divisions cellulaires.

L'hémigonadectomie induit une hypertrophie compensatrice du testicule restant quand elle est pratiquée soit chez le Rat impubère ou prépubère (WIJNANS, I953 ; Jacobsohn et Norgren, I965; Eguch et Morikawa, ig68; Figarova, ig69; 
LeVina, I97 ; Yagrixuma et al., i969) soit chez le Béliet adulte (Voglamayr et MATTNER, I968; SKINNER, I97 i JOHNSON et al., I97I). L'algnientation du poids testiculaire peut être reliée à l'accroissenlent de l'efficacité de la spermatogenèse. Nous avons donc analysé chez des animaux adultes les stocks de spermatogonies souches et de cellules de Sertoli, les rendements des divisions cellulaires au cours de la spermatogenèse et la production quotidienne de spermatides après hémicastration soit de rats âgés de I à Io jours, soit de béliers adultes présentant des variations saisonnières de l'activité sexuelle.

\section{MA'TÉRIEL ET MÉTHODES}

Des rats Wistar de souche o3 INIRA ont été hémicastrés à l'âge de I,5 ou 1o jours. lls ont été abattus en même temps que des animaux témoins (3 animaux/lot).

Des béliers Ile-de-France adultes, soumis aux conditions saisonnières naturelles ont été hémicastrés en mars (9 animaux) ou en octobre (3 animaux). Le deuxième testicule a été prélevé 6 mois plus tard.

Les testicules ont été pesés et traités pour l'histologie classique (Hochereav, i967).

Les volumes relatifs des tubes séminifères et du tissu intertubulaire ont été déterminés à l'aide d'un oculaire intégrateur Zeiss sur 40 champs oculaires pour chaque testicule. Les volumes totaux des tubes séminifères et du tissu intertubulaire ont ensuite été calculés.

Les diamètres des tubes séminifères ont été mesurés à l'aide d'un micromètre oculaire sur 20 sections orthogonales par testicule. La longueur totale des tubes séminifères par testicule a été calculée à partir de la formule de Atral et Courot (1963).

La classification des stades du cycle de l'épithélium séminifère utilisée est celle de RoOsENRunge et Giesel (I950) et Ortavant (I958). Dix sections orthogonales (de io $\mu$ d'épaisseur) de tubes séminifères ont été analysées pour chaque stade étudié.

Dans les testicules de rat, les noyaux des cellules de Sertoli, des spermatogonies A souches, des spermatocytes I préleptotène et des spermatides rondes ont été dénombrés au début du stade 8.

Dans les testicules de bélier, les noyaux des spermatogonies $A_{0}$ et $A_{1}$, spermatogonies souches respectivement de réserve et cycliques selon la nomenclature de CLERMont (I967) et des cellules de Sertoli ont été comptées respectivement aux stades 7 , 8 et $\mathbf{1}$. I.es spermatocytes I leptotène et les spermatides rondes ont été dénombrées respectivement aux stades 2 et I

Les nombres moyens de noyaux de cellules germinales par section orthogonale de tubes séminifères de ro $\mu$ d'épaisseur ont été corrigés en fonction du diamètre nucléaire de chaque catégorie cellulaire et de l'épaisseur de coupes, selon OrTAVANT (I958).

Les nombres totaux de cellules de Sertoli et de spermatogonies souches par testicule ont ensuite été calculés à partir de la longueur totale des tubes séminifères et du nombre de cellules par coupe. Le rendement des divisions spermatogoniales a été évalué à partir du rapport entre le nombre de spermatocytes 1 préleptotène ou leptotène et le nombre de spermatogonies $A$ souches (rat) et $A_{1}$ (bélier). Le rapport entre le nombre de spermatides rondes (stades 8 ou $\mathrm{r}$ ) et le nombre de spermatocytes I préleptotène ou leptotène correspond au rendement global de la prophase méiotique, des divisions méiotiques et du début de la spermiogenèse.

La production quotidienne de spermatides rondes a été cléterminéc selon la formule de AMAN et Almouist (1962).

La surface des noyaux des cellules de Sertoli, des spermatogonies souches, des spermatocytes I et des spermatides rondes a été évaluée à partir des mesures de 20 noyaux par type cellulaire, dessinés à la chambre claire

La fertilité des rats hémicastrés ì l'âge de 5 jours a été analysée à l'àcé de I 35 jours en mettant chaque mâle avec 3 femelles penclant 6 jours consécutifs. Ic nombre de femelles gestantes et le nombre de petits par portée ont été déterminés. Teur fertilité a été comparée à celle de rats normaux.

Les animaux ont été abattus le $\tau^{\circ}$ jour ct le tractus génital prélevé et pesé. Au moment de l'abattage, le sang a été prélevé, sous anesthésie à l'éther, et la LH cléterminée par dosage radioimmunologique selon Viguier-.Martinez et Pelletier (igj2).

Chez le Bélier, le sang a été collecté chez 9 des animaux normaux et 9 animaux hémicastrés par ponction dans la veine jugulaire, matin et soir, deux jours avant l'hémicastration puis tous les quinze jours de mars à octobre. Le dosage de I.H a été effectué selon la technique de PeLLETIER et al., (1968). 


\section{RÉSULTATS}

\section{A. - Poids testiculairo}

L'hémicastration entraîne chez le Rat adulte une hypertrophie pondérale significative du testicule restant de +44 p. roo, +44 p. Ioo et +2 I p. Ioo respectivement après hémigonadectomie à $I, 5$ ou Io jours d'âge (tabl. I).

\section{TABLEAU I}

Effet de l'hémicastration pratiquée chcz le rat impubèré, sur le testicule restant prélevé à l'âge de 120 jours

(3 animaux/lot; $m \pm s m$ )

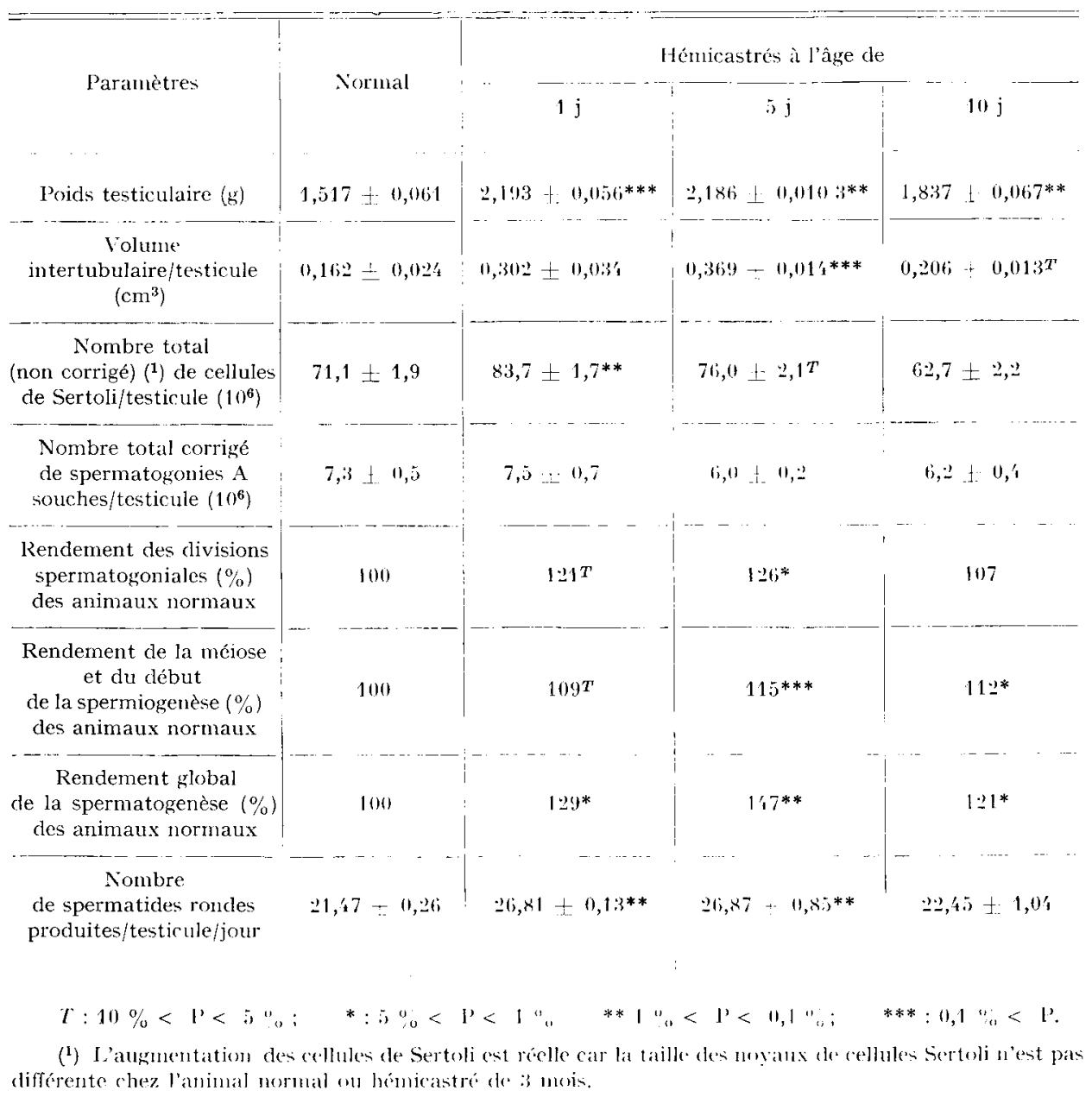


Chez le Bélier adulte, le testicule restant après hémicastration est toujours significativement plus lourd que celui des animaux normaux pris à la même saison, respectivement de $+3 \mathrm{I}$ p. Ioo et $+44 \mathrm{p}$. Ioo au printemps et à l'automne (tabl. 2). Cependant chez les animaux hémicastrés à l'automne et prélevés au printemps suivant le poids testiculaire de départ est seulement maintenu.

\section{B. -- Les cellules de Sertoli}

L'hémicastration peut modifier les cellules de Sertoli, au moins dans certaines conditions.

Ainsi, l'hémigonadectomie pratiquée chez le Rat âgé de I jour entraîne chez le Rat adulte une augmentation significative du nombre total de cellules de Sertoli ( + I7 p. Ioo) (tabl. I) dans le testicule restant; cette augmentation est réelle car il n'y a pas de modification de la taille nucléaire des cellules de Sertoli dans le testicule restant.

Par ailleurs, chez le Bélier adulte, 1'hémicastration provoque une augmentation $(+33$ p. I00) significative de la taille nucléaire des cellules de Sertoli chez les animaux hémicastrés à l'automne et prélevé au printemps suivant (tabl. 2), mais le nombre total n'est pas modifié, ces cellules ne se multipliant pas chez l'adulte.

TABI,EAU 2

Effet de l'hémicastration chez le Béliev Ile de France adulte

\begin{tabular}{|c|c|c|c|c|}
\hline \multirow[b]{2}{*}{ Paramètres } & \multicolumn{2}{|c|}{ Printemps } & \multicolumn{2}{|c|}{ Automne } \\
\hline & Entier & $\begin{array}{l}\text { Hémicastré } \\
6 \text { mois plus tôt }\end{array}$ & Entier & $\begin{array}{l}\text { Hémicastré } \\
6 \text { mois plus tôt }\end{array}$ \\
\hline Poids testiculaire $(g)$ & $182 \pm 2 t$ & $2 \cdot 40 \pm 9$ & $247 \pm 12$ & $356 \pm 93 * * * a$ \\
\hline $\begin{array}{c}\text { Surface nucléaire } \\
\text { cellule de Sertoli }\left(\mu \mathrm{m}^{2}\right)\end{array}$ & $47,6 \pm 0,9$ & $6,3,4 \pm 1,5 * * *$ & $56, \bar{j} \div 1,1$ & $57,9 \div 1,0$ \\
\hline $\begin{array}{l}\text { Spermatogonies } \\
\mathrm{A}_{0} / \text { testicule }\left(10^{6}\right)\end{array}$ & $252 \pm 50$ & $515 \pm 120^{*}$ & $530 \pm 161$ & $775 \pm 108$ \\
\hline $\begin{array}{l}\text { Spermatogonies } \\
\text { A } 1 \text { testicule }\left(10^{6}\right\}\end{array}$ & $343 \pm 3^{\prime}$ & $572 \pm 56 * * *$ & $558 \div 60$ & $781 \pm 82 *$ \\
\hline $\begin{array}{c}\text { Spermatocytes } \\
\text { I Leptotène/sperma- } \\
\text { togonie } A_{1}\end{array}$ & $19,5 \pm 2,2$ & $17,9 \div 1,2$ & $26,3 \pm 1,8$ & $28,5 \pm 2,0$ \\
\hline $\begin{array}{l}\text { Spermatides rondes/ } \\
\text { spermatocytes I lept. }\end{array}$ & $2,8 \pm 0,2$ & $3,4 \pm 0,3^{b}$ & 3,0 上 0,1 & $2,8 \pm 0,1$ \\
\hline $\begin{array}{l}\text { Production quotidienne } \\
\text { de spermatides } \\
\text { rondes/testicule }\left(10^{9}\right)\end{array}$ & $1,9 \pm 0,3$ & $3,3 \pm 0,3^{* *}$ & $3,9 \pm 0,3$ & $5,7 \pm 0,5^{* *}$ \\
\hline
\end{tabular}

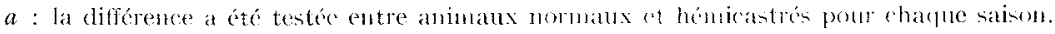

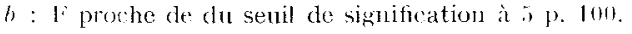




\section{C. - Les spermatogonies souches}

I a réponse des spermatogonies souches à 1'hémicastration diffère entre les deux espèces.

Pratiquée à l'âge de I jour chez le Rat, l'hémigonadectomie n'entraîne pas de modification du nombre des spermatogonies souches tandis que, effectuée à l'âge de 5 ou Io jours, elle provoque une diminution non significative ( 8 et 16 p. Ioo respectivement) de ce nombre (tabl. I).

Par contre, chez le Bélier, l'hémigonadectomie provoque une augmentation significative des spermatogonies $A_{0}$ et $A_{1}$, par rapport aux animaux normaux prélevés à la même saison (tab1. 2).

\section{D. - Les divisions cellulaires au cours de la spermatogenèse}

L'ablation d'un testicule chez le Rat de 5 jours augmente significativement ( +26 p. IOo) le rendement des divisions spermatogoniales (tab1. I).

Le rendement de l'ensemble des processus comprenant la prophase méiotique, les divisions méiotiques et le début de la spermiogenèse est augmenté significativement chez le Rat hémicastré à 5 ( + I 5 p. IOO) ou Io ( + I2 p. IOo) jours. L'efficacité de l'ensemble des processus compris entre les divisions de spermatogonies A souches et les spermatides rondes est alors significativement améliorée de respectivement 29,47 et 2 I p. Ioo pour des animaux hémicastrés à l'âge de I, 5 ou ro jours (tabl. I).

Chez le Bélier adulte, l'hémicastration n'entraîne pas, 6 mois plus tard, de modification dans le rendement des divisions spermatogoniales (tabl. 2). Le rendement de l'ensemble de la méiose et du début de la spermiogenèse est augmentée significativement de $2 \mathrm{I}$ p. Ioo, chez l'animal hémicastré et prélevé au printemps par rapport aux animaux témoins pris à la même saison (tabl. 2).

\section{E. - Production quotidienne de gamètes}

De l'ensemble des modifications (nombre de spermatogonies souches ; rendement des divisions cellulaires), il découle que chez le Rat hémicastré à l'âge de I ou 5 jours (respectivement +33 p. Ioo et +25 p. IOO), de même que chez la Bélier hémigonadectomisé prélevé au printemps ou à l'automne $\left(+73 \mathrm{p}\right.$. Ios et $+4^{6} \mathrm{p}$. Ioo respectivement), il y a augmentation significative de la production quotidienne des spermatides rondes.

\section{F. - Fertilité des rats hémicastrés}

Le pourcentage de femelles gestantes (73 p. Ioo) et le nombre de petits par portée $(8,7 \pm \mathrm{I}, 0)$ sont les mêmes chez le Rat hémicastré à l'âge de 5 jours et chez 1'animal adulte normal (respectivement $73 \pm 9$ p. Ioo et $8,7 \pm 0,8$ ).

\section{G. - Toneur plasmatique en hormone $L H$}

Chez le Rat adulte, on n'observe pas de différence significative de la teneur plasmatique en hormone LH entre les animaux normaux $(4, \mathrm{IO} \pm 0,6 \mathrm{ng} / \mathrm{ml})$ et les animaux hémicastrés à l'âge de 5 jours $(4,3 \pm 0,7 \mathrm{ng} / \mathrm{ml})$. 
Au contraire, chez le Bélier hémicastré au printemps, la quantité d'hormone LH circulant dans le sang augmente rapidement durant le $\mathrm{I}^{\text {er }}$ mois après hémigonadectomie et reste jusqu'à l'automne à un niveau plus élevé ( $\mathrm{I}, 70$ 土 0 , Io ng/ml) que chez les animaux normaux $(0,9 \pm 0,05 \mathrm{ng} / \mathrm{ml})$. Par ailleurs, on observe une corrélation positive $(r=0,49)$ significative $(P=0,05)$ entre la quantité d'hormone LH circulante et le rendement des divisions spermatogoniales que ce soit chez les animaux normaux ou les animaux hémicastrés.

\section{DISCUSSION ET CONCLUSION}

L'hémicastration pratiquée chez le Rat en croissance entraîne une hypertrophie compensatrice du testicule restant (WIJNANs, I953; JACOBSOHN et NoRGREN, I965. EGuchi et Morikawa, I968 ; Figarova, I969; LEvina, I97 I ; Yaginuma et al., ig69.) Cependant seuls les animaux hémicastrés au plus tard à l'âge de $\mathrm{I} 5$ jours développent une hypertrophie définitive du testicule restant (HOCHEREAU-de REVIERS, I97I). Ceci s'oppose aux résultats de NEGRI et WEBER (I949) et GRaNT (I956) et correspond à ce que nous avons par ailleurs obtenu chez le Veau et le Taureau hémicastrés à 4 mois et 4 ans (HocherEaU-de ReviERs I970).

Au contraire chez le Bélier adulte, l'hémicastration induit toujours une hypertrophie du testicule restant par rapport à celui d'animaux pris à la même saison (Voglmayr et Mattiner, I968; Skinner, I97I ; Johnson et al., I972). Un tel phénomène a déjà été observé chez des animaux présentant des variations saisonnières de 1'activité sexuelle (Lapin : RIBBERT, I890 ; EDWARDS, I940 ; Blaireau : CANIVENC et Relexans, I967 ; Campagnol : Martinet et Meunier, I975).

Dans les deux espèces étudiées, l'accroissement du poids testiculaire est dû à la fois à l'augmentation des tubes séminifères et du tissu intertubulaire. Ce dernier point avait déjà été observé par ANCEL et Bouin (I903), EGUCHI et MorikawA, I968 ; YaGinuma et al. (I969) Liang et Liang (I970) et SAN Filippo (I970). L'augmentation des tubes séminifères porte en partie sur un accroissement de la longueur des tubes séminifères qui serait relié à une élévation du niveau plasmatique de 1'hormone FSH (FIGarova, I969).

Les cellules de Sertoli répondent à l'hémicastration. Dans le cas de rats hémigonadectomisés à l'âge I ou 5 jours, elle provoque une augmentation du nombre total de cellules de Sertoli par testicule. Au moment de l'opération, les cellules de Sertoli se divisent activement (NAGY, I972 ; STEINGERGER et STEINBERGER, I97I) et l'arrêt de leurs mitoses se situe vers l'âge de $\mathrm{I}_{5}$ jours au moment où l'hémicastration ellemême n'a plus d'effet définitif.

Le nombre de ces cellules est fixé bien avant la puberté ; il est fonction de l'équilibre endocrinien (Couro'r, I97r).

Chez les animaux présentant des modifications saisonnières de l'activité sexuelle comme le Bélier, il existe des variations saisonnières de la taille nucléaire des cellules de Sertoli (Hamster : VENDREI,Y et al., I972). L'hémicastration élimine alors la régression saisonnière de ces cellules.

Aux variations des cellules de Sertoli peuvent être reliées au moins les variations de l'efficacité de la méiose et de la spermiogenèse car les spermatocytes et les sperma- 
tides ont des relations très étroites avec les cellules de Sertoli. Un système complexe de jonctions cellulaires isolent ces cellules de l'apport direct de substances venant de l'extérieur des tubes séminifères, qui doivent alors transiter par les cellules de Sertoli (DYM et FAwCETT, I97I).

La réponse du nombre total de spermatogonies souches à l'hémicastration est très différente dans les deux espèces étudiées.

Chez le Rat, on observe, soit un maintien au niveau normal, soit une légère diminution non significative du nombre total de spermatogonies souches par testicule.

Au contraire, chez le Bélier adulte, 1'ablation d'un testicule provoque une augmentation du stock de spermatogonies $A_{0}$ et $A_{1}$ dans le testicule restant. Dans cette espèce, le nombre de spermatogonies souches varie en fonction de la saison (ORTAVANT. I959). Ceci suppose donc que le stock de spermatogonies souches peut varier en fonction de l'équilibre endocrinien, au moins dans certaines conditions.

Le rendement des divisions spermatogoniales est en général très mauvais - 50 p. Ioo chez le Rat (Clermont et Bustos-Obregon, ig68), 28 et 58 p. Ioo chez le Bélier respectivement au printemps et à l'automne.

Il est amélioré significativement seulement après hémicastration à l'âge de 5 jours chez le Rat. Dans cette espèce, nous n'avons pas pu mettre en évidence de différence pour les niveaux plasmatiques de l'hormone $\mathrm{L}_{1} \mathrm{H}$ entrę hémicastrés et normaux à l'âge adulte et donc les relier aux modifications observées par ailleurs. Toutefois, la sensibilité du dosage dans cette espèce n'est pas très grande ; il est possible qu'une petite modification existe sans qu'elle apparaisse ici. Par ailleurs, elle a pu être seulement transitoire et ne plus exister ensuite.

Chez le Bélier, dans le cadre de cette expérience, il n'apparaît pas d'augmen-

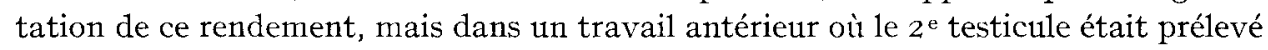
I, 2 ou 3 mois après l'hémicastration pratiquée au printemps ; nous avions constaté une augmentation de ce rendement dès le $\mathrm{I}^{\mathrm{er}}$ mois tandis que à l'automne, dans les mêmes conditions, la régression était retardée (HochereaU de REviers et PELIETIER, I97I). Par ailleurs, on a pu mettre en évidence une corrélation positive significative entre le rendement des divisions spermatogoniales et la teneur plasmatique en hormone $\mathrm{LH}$.

Le rendement de l'ensemble formé par la méïose, les divisions méïotiques et la spermiogenèse est amélioré significativement chez le Rat après hémicastration à l'âge de 5 ou ro jours. Chez le Bélier, la production de cette étape est essentiellement améliorée dans le cas de prélèvement au printemps. Ceci peut être rapproché de l'augmentation de la taille des noyaux de cellules de Sertoli. L'accroissement de l'efficacité de la spermatogenèse peut découler à la fois de l'augmentation des cellules de Sertoli (activité cellulaire ou nombre) et de la taille des tubes séminifères qui permettent d'accroître le nombre de cellules germinales en liaison avec chaque cellule de Sertoli.

De l'ensemble de ces modifications découle, dans les deux espèces, une augmentation de la production quantitative de spermatides rondes par testicule et par jour.

Des résultats assez contradictoires sur la production et la récolte quotidienne de sperme ont été obtenus chez des rats hémicastrés à l'âge adulte (SMEI,SER, I933 ; MaUss et HackstedT, I972).

La fertilité des femelles saillies par des animaux hémicastrés à l'âge de 5 jourss 
et leur nombre de petits par portée ne sont pas différents de celles saillies par des animaux normaux. La qualité des spermatozoïdes prođuits par un rat hémicastré à l'âge de 5 jours n'est donc probablement pas inférieure à la normale, les glandes annexes étant maintenues au même niveau que chez l'animal normal. On a donc amélioration de la quantité de spermatozoïdes produit par testicule avec une qualité au moins égale à la normale.

Chez le Bélier, nous n'avons pas pratiqué d'analyse de la qualité du sperme produit. LoIR (I974) avait observé une légère augmentation proche de la signification de la taille cellulaire des spermatocytes pachytène entre béliers normaux ( $\left.2336 \pm 44 \mu^{3}\right)$ et hémicastrés $\left(2428 \pm 295 \iota^{3}\right.$ ) prélevés au printemps. Il est possible que ceci soit lié à une amélioration qualitative du gamète mâle prođuit. Rien ne permet de 1'affirmer actuellement et ceci reste à analyser.

Nous pouvons conclure à partir de cette étude utilisant l'hémicastration comme technique qu'il est, théoriquement, possible chez l'animal normal, en agissant soit avant la puberté - comme chez le Rat - , soit chez l'adulte — en particulier dans le cas d'animaux présentant des variations saisonnières de l'activité sexuelle, d'obtenir une amélioration de l'efficacité de la spermatogenèse. En ce cas, elle est liée non seulement aux cellules germinales elles-mêmes dont les dégénérescences sont diminuées, mais aussi aux cellules de Sertoli.

Les mécanismes mis en évidence dans le cas de 1'hypertrophie compensatrice peuvent agir aussi chez l'animal normal. En premier lieu ceci peut simplement être dû à la modification des androgènes testiculaires diminués au moins temporairement après l'hémicastration, qui provoquerait une augmentation de la sécrétion de $\mathrm{L}_{\mathrm{H}} \mathrm{H}$ (ou aussi de FSH). Toutefois, les tubes séminifères eux-mêmes peuvent être mis en jeu ; ceci apparaît au moins chez le Bélier après hémicastration au printemps. Par ailleurs, la quantité d'inhibine sécrétée par les cellules de Sertoli dans le fluide testiculaire et que module la sécrétion de FSH diminue après hémicastration; il y aurait alors augmentation de la sécrétion de FSH. Ceci a effectivement été observé après hémicastration chez le Rat prépubère par Frgarova (I969) JoHnson (I969) et par OJEDA et RAMIREZ (I972).

Enfin, les cellules germinales elles-mêmes pourraient contrôler la sécrétion de FSH par une "gonocytine "; cette dernière explication a été avancée par I,EVINA (I97I).

Il est en fait probable que chacun des facteurs concernés agit directement ou indirectement dans le contrôle de l'efficacité de la spermatogenèse.

Colloque D. G.R. S. T., Biologie de la Procréation,

Paris 7-8 mars 1975 .

\section{REMERCIEMENTS}

I.es dosages plasmatiques de la. LH plasmatique chez le Rat et le Bélier hémicastrés ont été effectués respectivement par Mme M. C. Viguier-Martinez (Faculté des Sciences, Tours) et par J. Pelletier (1. N. R. A., Nouzilly); qu'ils en soient ici remerciés.

Ce travail a été réalisé avec l'aide des contrats D. (. R. S. T. no $12-7$-oog8 et 7f-7-0038. 


\section{SUMMARY}

\section{INCREASE OF SPERMATOGENESIS EFFICIENCY}

\section{BY HEMICASTRATION IN RAT AND RAM}

The efficiency of spermatogenesis is drastically diminished by cellular degeneration occurring especially during spermatogonial divisions. We have tried to counteract this degeneration by compensatory hypertrophy of the testis following hemicastration.

Wistar rats were hemicastrated when they were I, 5 or ro days old. Control and experimental animals were sacrificed at I $20-130$ days of age.

Adult Ile-de-France rams, living under natural conditions, were hemicastrated either in march or in october. The remaining testis was removed 6 months later.

Sertoli cells, A stem spermatogonia, primary leptotene spermatocytes and round spermatids were counted in round cross sections of seminiferous tubules and their corrected number per cross section calculated (ORTAVANT, I958). The total number of Sertoli cells and stem spermatogonia was estimated. The yield of spermatogonial divisions and of the step involving meiotic prophase, meiotic divisions and the beginning of spermatogenesis were analysed. The daily production of round spermatids was also determined (AMANN and ALmoUIST, 1962).

The surface of Sertoli cell nuclei was measured in hemicastrated and normal rats and rams.

Adult rats hemicastrated when 5 days old were mated with 3 females for 6 days. The number of pregnant females and the number of pups per litter were compared to that of normal adult rats. Plasma levels of LH of these hemicastrated and normal animals were determined by the method of Viguier-Martinez and Pelletier (I972).

Normal and hemicastrated rams were bled twice a day each fortnight from march to october. Plasma LH levels were determined by the method of Pelletier et al. (I968).

The remaining testis had developed a true hypertrophy either after hemicastration in the impuberal or prepuberal rat (I day, +44 p. Ioo 5 days, +44 p. Ioo; Io days, +2 I p. Ioo), or in the adult ram (spring: $+32 \mathrm{p}$. Ioo; autumn : $+44 \mathrm{p}$. Ioo) as compared to the normal animal taken at the same season.

Sertoli cells were modified by hemicastration.

In $\mathbf{I}$ day hemicastrated rats, a significant increase in the total number of Sertoli cells per testis was obtained. In the adult ram an increase in nuclear size of Sertoli cells was observed in the remaining (spring) testis of rams hemicastrated in autumn.

Change in the stock (per testis) of stem spermatogonia were different between the two species.

In 5 or Io day old rats, hemicastration was associated with a diminution in the total number of stem spermatogonia, as compared with normal or I day hemicastrated animals.

But in the hemicastrated adult ram there was always an increase in the stock per testis of stem spermatogonia as compared with intact animals investigated at the same season.

Efficiency of spermatogenesis was increased by hemicastration. The yield of spermatogonial divisions was significantly increased in rats hemicastrated at 5 days.

The yield of the whole process, including meiosis and the beginning of spermiogenesis was increased by hemicastration in rats hemicastrated at either 5 or Io days, as well as in the remaining testis of rams hemicastrated in the autumn whose remaining testis was removed for examination 6 months later.

We have observed a significant increase in the daily production of round spermatids in the two species.

The fertility of normal rats and those hemicastrated at 5 days was equal.

No significant difference was observed between the plasma LH level of normal adult rats and adult rats hemicastrated before puberty.

In the adult ram, the plasma LH level had increased significantly after hemicastration performed in spring. It was maintained higher until october. A positive correlation $(+0.49$, $\mathrm{P}=0.05$ ) was observed between individual plasma $\mathrm{LH}$ values and the yield of spermatogonial divisions.

By means of hemicastration, we have obtained in the two species an increase in the efficiency of spermatogenesis. The role of Sertoli cells is discussed. 


\section{RÉFÉRENCES BIBLIOGRAPHIQUES}

Ancel P., Bouin P., I903. Recherches sur le rôle de la glande interstitielle du testicule. Hypertrophie compensatrice expérimentale. C. R. Acad. Sc. Paris, Sér. D, 137, 1288-1289.

Amann R. P., Almovist J. O., rg62. Reproductive capacity of dairy bulls. VIII. Direct and indirect measurement of testicular sperm production. J. Dairy Sci., 45, 774-78I

Attal J., Courot M., Ig63. Développement testiculaire et établissement de la spermatogenèse chez le Taureau. Ann. Biol. anim. Bioch. Biophys., 3, 2I9-24I.

Canivenc R., Relexans M. C., I967. Résultats de l'hémicastration chez le Blaireau mâle (Meles Meles). C. R. Acad. Sc., Paris, Ser. D, 264, $2138-2140$.

Clermont Y., I967. Cinétique de la spermatogenèse chez les Mammifères. Arch. Anat. Microsc. Morph. exp., 56, (Suppl. 3-4), 7-60.

Clermont Y., Bustos-Obregon E., I968. Re-examination of spermatogonial renewal in the rat by means of seminiferous tubules mounted in toto. Am. J. Anat., 122, $237 \cdot 24.8$.

Courot M., I97I. Établissement de la spermatogenèse chez l'agneau (ovis aries). Étude expérimentale de son contrôle gonadotrope ; importance des cellules de la lignée sertolienne. Thèse Doct. Sci., Paris, C.N.R.S., $\mathrm{AO} 6317$.

DYm M., FAwCETT D. W., I97I. Further observations on the numbers of spermatogonia, spermatocytes and spermatids connected by intercellular bridges in mammalian testis. Biol. Reprod., 4, $195^{-215}$.

Edwards J., 1940. The effect of unilateral castration on spermatogenesis. Proc. Roy. Soc., Lond., Ser. $B, 128,407$.

Eguchi Y., MorikawA Y., r968. Changes in pituitary gonadal interrelations during perinatal days in the rat. Anat. Record, 161, I63-I 70.

Figarova V., I969. Influence of hemicastration on morphological gonade structure and development of spermiogenic epithelium in male infantile rats. Folia Morphol., 17, 348-354.

Grant J. H., I956. IV. The effects of unilateral orchidectomy on the rat testis. Proc. Soc. Study Fert., 8, 27-35.

Hochereau M. T., T967. Synthèse de l'ADN au cours des multiplications et du renouvellement des spermatogonies chez le Taureau. Arch. Anat. Microsc. Morph. exp., 56 (suppl. 3-4), 85-96́.

Hochereau de Reviers M. T., r97o. Eitude des divisions spermatogoniales et du venouvellement de la spermatogonie souche chez le Taureau. Thèse Doct. Sci., Paris, C.N.R.S., AO 3976.

Hochereau de Reviers M. T., I97I. Action de l'hémicastration sur l'évolution du testicule restant chez le Rat. Bull. Assoc. Anat., 151, 364-371.

Hochereau de Reviers M. T., Pelletier J., i97i. Unilateral castration in the ram. J. Reprod. Fert., 27, 498, (abstr.)

J AcoBsohn D., Norgren A., 1965. Early effects of testosterone propionate injected into 5 days old rats. Acta Endocr., 49, 453-465.

Johnson D. C., I969. Elevation of plasma gonadotrophins in unilaterally castrated male rats. J. Endocr., 43, 3II-3I 2 .

Johnson B. H., Desjardins C., Ewing L. L., I972. Testicular function following hemicastration in mature rams. J. Anim. Sci., 33, 257.

Levina S. E., I97I. Eiffects of hemicastration in infantile rat. VI ${ }^{\mathrm{e}}$ C. E. E. C., Montpellier, Abstr. I22.

Liang D. S., LiAng M. D., I970. Testicular hypertrophy in rats. J. Reprod. Fert., 21, 537-540.

LoIR M, I974. Communication personnelle.

Martinet L., Meunier M., I975. Rôle de la durée quotidienne d'éclairement sur la réponse, après hémicastration du testicule restant chez le campagnol des champs (Micronus arvalis). Ann. Biol. anim. Bioch. Biophys., 15, (sous presse).

Mauss J., Hackstedt G., I972. The effect of unilateral orchidectomy and unilateral cryptorchidism on sperm out put in the rat. J. Reprod. Fert., 30, 289-292.

NAGY F., r972. Cell division kinetics and DNA synthesis in the immature Sertoli cells of the rat testis. J. Reprod. Fert, 28, 389-395.

Negri L., WEBER G., I949. Applicazione di metodi isto-biometrici allo studio della iperattivita funzionale testicolare dopo emicastrazione. Archivo de Veschi Anat. Pat., 13, 469-487.

OJEDA S. R., Ramirez V. D., 1972. Plasma level of LH and FSH in maturing rats : response to hemigonadectomy. Endocrinology, 90, 466-472.

Ortavant R., 1958. Le cycle spermatogênétique chez le Bélier. Thèse Doct. ès Sci., Paris.

Ortavant R., I959. Spermatogenesis and morphology of the spermatozoon. In Reproduction in Domestic Animals, Ist Ed., Cole et Cupps Ed., Acad. Press Publ., N. Y., vol II.

Pelletier J., Kann G., Dolais J., Rossein G., i 968 . Dosage radioimmunologique de l'hormone lutéinisante plasmatique chez le Mouton. Mise au point de la technique de dosage. C. R. Acad. Sci., Paris, Ser, D, 266, 2291-2294. 
RibBert H., I89o. Ueber die compensatorische Hypertrophie der Geschlechtsdrüsen. Virchows Archiv., 120, 247-272.

Roosen-Runge E. C., Giesel L. O., r950. Quantitative studies on spermatogenesis in the Albino rat. Amer. J. Anat., 87, I-30.

SKinner J. D., I97r. Compensatory testicular hypertrophy and hypertrophy of the homolateral ampulla following unilateral orchidectomy in the adult ram. Agroanimalia, 3, 97-98.

Smelser G. K., 1933. Compensatory hyper-activity of the testis following unilateral castration. Anat. Record., 57, Suppl. I, 28-29.

SANFilippo S., I970. Ultrastrukturelle untersuchungen an den Leydigschen Zwischenzellen des Rattenhodens nach einseitiger Gonadektomie. Endokrinol., 55, 270-285.

Steinberger E., I97I. Hormonal control of mammalian spermatogenesis. Physiol. Rev., 51, r-22.

Steinberger A., Steinberger E., rg7i. Replication pattern of Sertoli cells in maturing rat testis in vivo and in organ culture. Biol. Reprod., I, 84-87.

Viguier-Martinez M. C., Pelletier J., i972. Étude comparée des effets de la cyprotérone et de l'acétate de cyprotérone sur la LH hypophysaire, la LH plasmatique et l'appareil génital du rat mâle prépubère. C. R, Acad. Sc., Paris, Ser. D, 274, 2696-2699.

Vendrely E., Guerillot C., Da Lage C., r972. Variations saisonnières des cellules de Sertoli et de Leydig dans le testicule du hamster doré. Étude caryométrique. C. R. Acad. Sc., Paris, Ser. D, 275, 4343-II46.

Voglmayr J. K., Mattner P. E., I968, Compensatory hypertrophy in the remaining testis following unilateral orchidectomy in the adult ram. J. Reprod. Fert., 17, I79-I8r.

Wijnans M., I953. The role of the nervous system in the development of compensatory testicular growth after hemicastration. Acta Endocrinol., 13, 69-73.

Yaginuma T., Kigawa T., Kobayashi T., ig69. Further studies on the hypothalamopituitaty testicular axis of the rat in the neonatal period. J. Endocr., 45, 473-474. 